\title{
Impact of electro-neuro-feedback on postoperative outcome of impacted lower third molar surgery
}

\author{
Giacomo Oteri ${ }^{1}$, Michele Pisano $^{1}$, Antonia Marcianò ${ }^{1}$, Gabriele Cervino $^{1}$, Matteo Peditto ${ }^{1}$
}

Correspondence: Dr. Matteo Peditto

Email: matteopeditto@gmail.com
'Department of Biomedical and Dental Sciences and Morphofunctional Imaging, University of Messina, Policlinic "Gaetano Martino", Messina, Italy

\section{ABSTRACT}

Objective: The purpose of this study was to evaluate the impact of a treatment with electro-neuro-feedback (ENF), a portable transcutaneous electrical nerve stimulation (TENS) device, on the clinical outcome and inflammatory biochemical parameters related to the impacted lower third molar surgery. Materials and Methods: A randomized, split-mouth, and single-blind study was conducted on 32 patients requiring lower third molars extractions and referred to the Oral Surgery Unit of the School of Dentistry of the University of Messina. Every patient underwent surgical removal of both lower third molars. The first extraction included a placebo (electrodes placement with turned-off device) treatment following the surgery, while the second had the ENF used next to the avulsion and the following days. Clinical parameters were collected 4 days before, immediately after, 2, 4, and 7 days after the surgical procedure. Biochemical parameters were obtained 1 day before the surgical removal and 7 days after. Data were processed using Wilcoxon-Mann-Whitney test, with significance being set at $P<0.05$. Results: Clinical outcome parameters showed a significant improvement after the ENF treatment, while considered inflammatory markers expressed different patterns. Conclusions: ENF, a reliable portable TENS device, has demonstrated to be a useful tool in the managing of the postsurgical phases, reducing edema, pain, and consequent pain-killers consumption.

Key words: Edema, electro-neuro-feedback, pain, third molar surgery, transcutaneous nerve stimulation

\section{INTRODUCTION}

"Electro-neuro-feedback (ENF)" is an electromedical device used in the physiotherapeutic treatments. Its functioning is based on the transcutaneous electrical nerve stimulation (TENS) principles. TENS refers to any electrical stimulation method that uses skin surface electrodes. ${ }^{[1]}$

Although the term comprehends a variety of devices and techniques, they all share a noninvasive, nonpharmacological aspect in the treatment of pain conditions. ${ }^{[2,3]}$ Stimulating the affected region with a low-voltage electrical current and skin electrodes, TENS

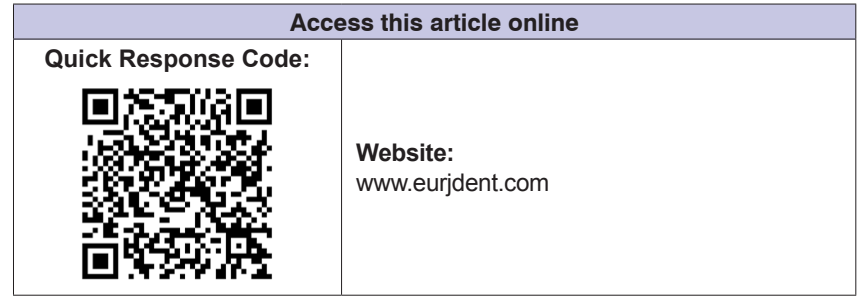

may inhibit the pain perception acting upon a "gating" mechanism in the dorsal horn of the spinal cord. ${ }^{[4,5]}$

According to the Gate Control Theory of Pain, this "gate" modulates the nociception intensity through small diameter afferent nerve fibers; whenever large diameter afferent nerve fibers receive some sort of stimulus, such as TENS, they can shut the "gate," thus lowering the pain perception.

Thanks to its versatility, TENS is widely used in different fields of medicine. ${ }^{[6-11]}$

This is an open access article distributed under the terms of the Creative Commons Attribution-NonCommercial-ShareAlike 3.0 License, which allows others to remix, tweak, and build upon the work non-commercially, as long as the author is credited and the new creations are licensed under the identical terms.

For reprints contact: reprints@medknow.com

How to cite this article: Oteri G, Pisano M, Marcianò A, Cervino G, Peditto M. Impact of electro-neuro-feedback on postoperative outcome of impacted lower third molar surgery. Eur J Dent 2018;12:77-88.

DOI: 10.4103/ejd.ejd_296_17 
ENF can record the impedance values of the human skin and to generate asymmetrical biphasic modified square wave pulses, according to a specific algorithm, for therapeutic purposes, with a real-time modulation of the current thanks to a negative feedback loop. Having no net DC component, eventual skin reactions are an unlikely occurrence.

Generating electrical patterns similar to the nerve pulses, ENF should provide relief for both acute and chronic pain. Therefore, considering that oral surgical interventions often lead to patient discomfort due to both edema and pain, it would be of clinical interest to evaluate the role exerted by this kind of treatment on the postop.

Lower third molar impaction is a condition frequently observed during dental practice. Many theories have been reported in the literature to explain the etiology and pathogenesis of atopic lower third molars. In the cases characterized by symptoms, associated pathology or also when the patient is requiring tooth removal, the treatment of choice is surgery using intra- or in third molar localized in an uncommon anatomical position the extraoral approach.

The surgical extraction of lower third molars is commonly followed by pain, swelling, and mouth functional limitation. ${ }^{[12-17]}$

Impacted lower third molars, when diagnosed with a similar anatomic position (in the respect of Pell and Gregory classification), represent an ideal scenario for split-mouth protocols, widely validated by literature. ${ }^{[18]}$

The purpose of this study was to observe the impact of the treatment with ENF on the patient-related outcome of the impacted lower third molars surgical removal. In addition to the pain-relieving action, secondary end-point of the study was to verify an eventual impact on the patient inflammatory status in the postop, analyzing both clinical and biochemical parameters.

\section{MATERIALS AND METHODS}

A randomized, split-mouth, and single-blind study was conducted at the Oral Surgical Unit, School of Dentistry, University of Messina during a 12-month (February 2016-January 2017) period. Patients aged between 18- and 30-year-old, without smoking habit, implantable electrical devices (e.g., cardiac pacemaker, cardioverter defibrillator), pregnancy, and health impairing conditions, were referred because requiring impacted lower third molars extractions. All impacted teeth were symmetrical and in Pell and Gregory Class II B and II C [Figure 1]. The indications of the extractions were pericoronal tissue infections, decayed teeth, and orthodontic reasons.

Thirty-two patients underwent a treatment protocol including two surgical lower third molar extractions and a single cycle of treatment with ENF following one of the avulsions.

Patients gave their consent to the treatment and were informed that their data would be used for statistical analysis related to this study. The prospective study was performed in accordance with the principles stated in the Declaration of Helsinki and the Good Clinical Practice Guidelines; informed consent was obtained from all participants.

The teeth were treated in two different steps, separated by a 2-month time interval, accordingly to a validated scheme. ${ }^{[19]}$ The first step included a placebo treatment (electrodes placement with turned-off device) following the surgery, while the second had the ENF used next to the avulsion. Each step was conducted accordingly to the following scheme:

- T0: clinical parameters assessment (cheek edema, mouth opening), blood samples collection for biochemical analyses;

- T1 (4 days after T0): surgical procedure, treatment with placebo/ENF

- T2 (6 days after T0): clinical parameters assessment (cheek edema, mouth opening), treatment with placebo/ENF

- T3 (8 days after T0): clinical parameters assessment (cheek edema, mouth opening), treatment with placebo/ENF

- T4 (11 days after T0): suture removal, clinical parameters assessment (cheek edema, mouth opening).

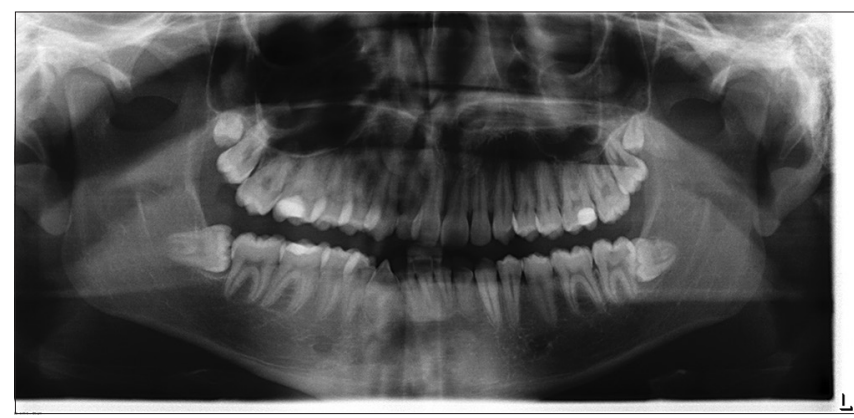

Figure 1: Dental Rx showing the symmetrical impaction of lower third molars 
All surgeries were performed by a single experienced oral surgeon. Preliminary to the reflection of a full-thickness 4-cornered mucoperiosteal flap, infiltration alveolar nerve block was performed. To gain sufficient access, eventual bone removal was considered, using a drill and cold sterile saline irrigation [Figure 2]. The extractions were performed with tooth sectioning, executed with a drill, and fragments removal was obtained by the use of levers. Following the extraction, curettage, and irrigation of the socket were performed. Wound closure was obtained through simple interrupted stitch with a 5.0 size nylon suture (Ethilon1, Ethicon Inc., Somerville, NJ) removed 7 days later.

Clinical parameters assessment pain intensity assessed by the patient using a visual analog scale (VAS), graduated from 0 to 10 . Schedule: Every $12 \mathrm{~h}$ for 7 days after the surgical procedure. Cheek Edema

Determined by measurements obtained with a tape measure: Tragus-labial commissure, tragus-pogonion, gonion-labial commissure, and gonion-pogonion [Figure 3]. Schedule: T0-T2-T3-T4.

Trismus and maximum mouth opening determined by measuring the maximum interincisal distance with a caliper (mm scale). Schedule: T0-T2-T3-T4.

Biochemical parameters assessment after collection, the blood samples were processed by the Central Laboratory of Clinical Pathology at the School of Medicine, University of Messina for determining the following values: inflammatory indexes: Time of erythrocyte sedimentation, C-reactive protein, Alpha-1-glycoprotein, interleukin (IL)-1-beta, IL-6, and tumor necrosis factor (TNF)-alpha.Schedule: T0-T4.

\section{Electroneuro-feedback treatment protocol}

ENF (FAST THERAPIES Via de Amicis, 33/3-25013 Carpenedolo (BS) ITALIA) treatment protocol was used according to the instructions of the manufacturer. The device emits electrical pulses with maximum current intensity of $20 \mathrm{~mA}$, duration of $3.3-483 \mathrm{~ms}$, frequency of $15.3-254 \mathrm{~Hz}$, and voltage of $20-450 \mathrm{v}$. Treatment requires a preliminary step, defined as "Scan," to identify the power level required for the treatment and the areas with the highest impedance; once located, the device adjusts its wavelength to optimize its interactions with the tissues.

After this step, two skin electrodes were placed between the labial commissure and the gonion [Figure 4], and the device was set for a $17^{\prime}$ long treatment, using

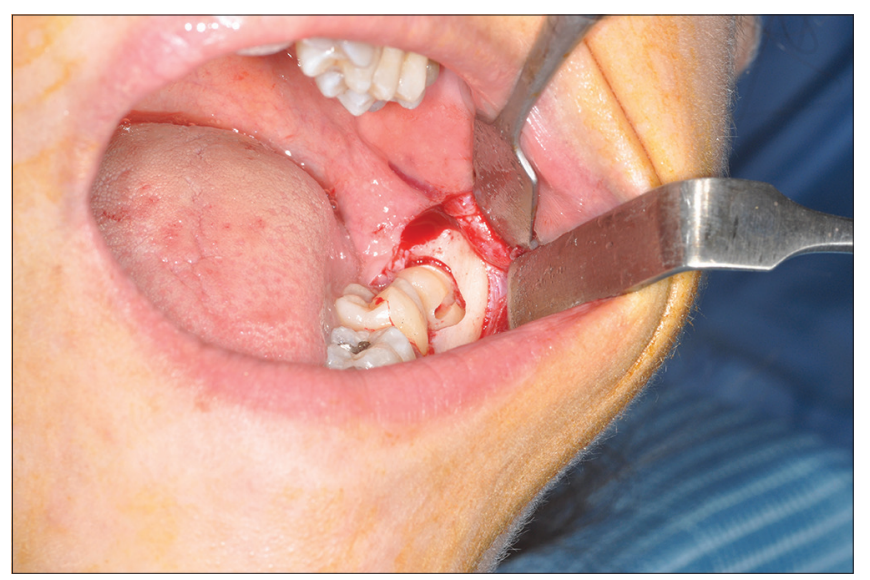

Figure 2: Intraoperative view of third molar surgery

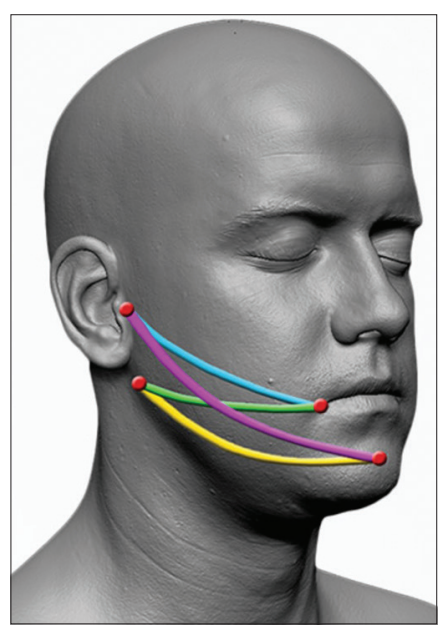

Figure 3: Facial measurements tragus-labial commissure (blue), tragus-pogonion (purple), gonion-labial commissure (green), gonion-pogonion (yellow)

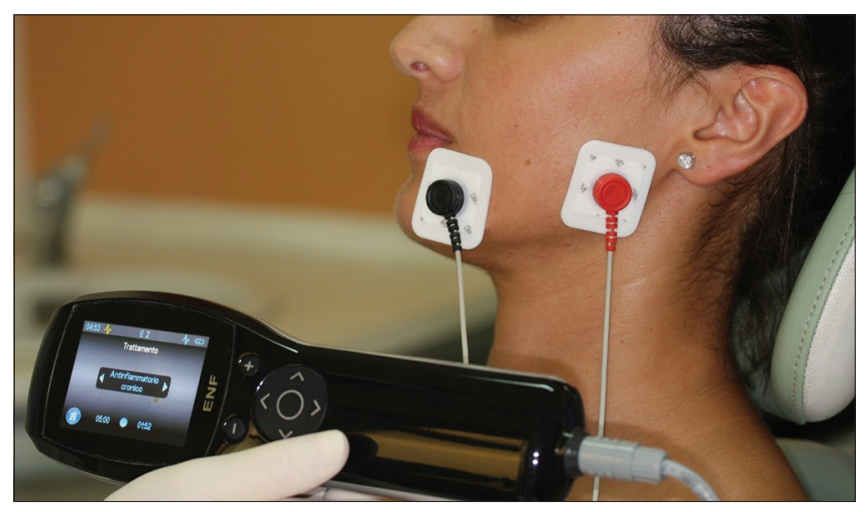

Figure 4: Operating electro-neuro-feedback and electrode placement

different manufacturer preset included in the software device.

Statistical Analysis Patients' clinical and biochemical measures were reported as a mean \pm standard deviation, and differences were assessed through relative change. Comparisons between groups at 
baseline and between baseline and follow-uP values at each time point were performed by Wilcoxon-MannWhitney test. Significance was set at $P=0.05$. MedCalc v15 (MedCalc Software bvba, Ostend, Belgium) was used for statistical calculations.

\section{RESULTS}

Among the considered sample, eight patients dropped out due to lack of the requested attendance. There were no adverse events following the surgical procedures. The treatment with ENF (test group) was safe in all patients. The clinical outcome parameters, percentage variations between baseline values and following times, and statistical analyses are represented in Tables 1-6. As per the test group, clinical parameters were significant improved at T1, T2, and T3 when compared to basal values. Perceived pain during the week following the surgical extraction was lower in the test, with statistical significance as per the first 5 days. As per the test group, no patient except one needed analgesic drugs the days after the surgery, while 20 out of 24 needed at least one dose in the control group. IL-1-beta, IL-6, and TNF-alpha values showed no significant variation between control and test groups. The improvement in the oral clinical conditions justified the oral procedure.

\section{DISCUSSION}

The TENS device was proved to be easy-to-use, with a safety profile. No adverse events related to the ENF occurred during the study.

Pertaining the primary outcome measures, following the surgical procedure, a significant number of patients showed a better clinical outcome concerning the pain-relieving action when ENF protocol was performed. In fact, using the VAS scale, significantly lower pain scores in the first 5 days after the surgery were observed in all test sites. The use of painkillers drugs was limited to a single event of the test group, against 20 events of the control group when at least a tablet of painkiller was administered.

In relation to the secondary outcome measures, the postsurgical swelling was less pronounced in the test group, and this result further supported the initial hypothesis that TENS treatment could lower the inflammatory response.

Anyway, no evidence was found in this sense, among the explored biochemical parameters.
In fact, it was found that control and test groups did not show a significant difference in terms of cytokines' levels; therefore, it is possible to assume that TENS does not play a role in the modulation of the inflammation, at least regarding the examined inflammatory marks. Edema consistent reduction may be explained through an interaction between the TENS and the muscular system; the muscle contractions induced by the treatment are supposed to enhance lymphatic and vascular mobility, thus lowering the fluid concentration in the extracellular environment. Patients showed a consistently better recovery regarding the functional limitation of the stomatognatic apparatus following the surgery, presumably for a combination of both edema and pain reduction.

This study confirmed the hypothesis that ENF can play a positive role, improving the patient related outcome when adopted after surgical removal of impacted third molars.

To the best of our knowledge, this study represents the first experience of investigation on the effect of ENF on the clinical outcome of the impact third molar surgery. This pain control method is widely applied in other fields of dentistry for the treatment of TMJ syndrome, trigeminal and postherpetic neuralgia, xerostomia periodontal pain or/as a coadiuvant for the intraoperative analgesia and many others. ${ }^{[19-23]}$

The question if ENF could improve oral clinical condition in postoperative was analyzed in a randomized, split-mouth, and single-blind study.

This initial research included a limited number of patients. Because of the size of our cohort of patients, the impact of ENF on postoperative clinical course should be further investigated to strengthen the collected data. Nevertheless, our data, next to the subjective evaluation of pain, explored some objective, measurable parameters such as edema and trismus, whose results are encouraging.

Recently, several methods have been explored to limit the postoperative effects of third molar surgery emphasizing the necessity for better pain, swelling, and trismus control. ${ }^{[24-27]}$

They include the use of drugs, the application of ice, low-power laser, the assumption of integrators. ${ }^{[19,28-30]}$

TENS is a pain-control method that uses low-voltage electrical current, applied to the skin through electrodes. 
Oteri, et al.: ENF outcome on impacted lower third molar surgery

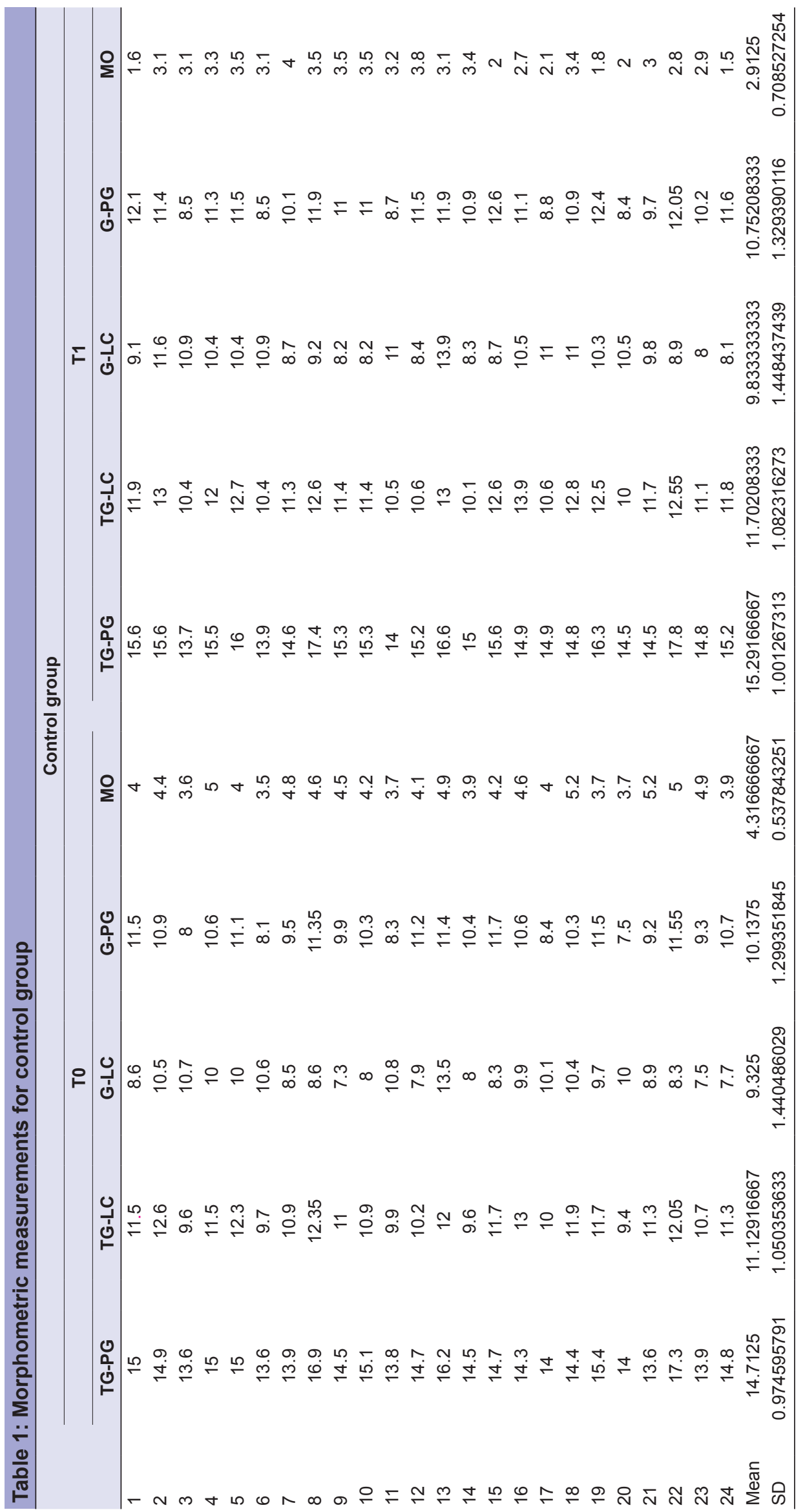


Oteri, et al.: ENF outcome on impacted lower third molar surgery

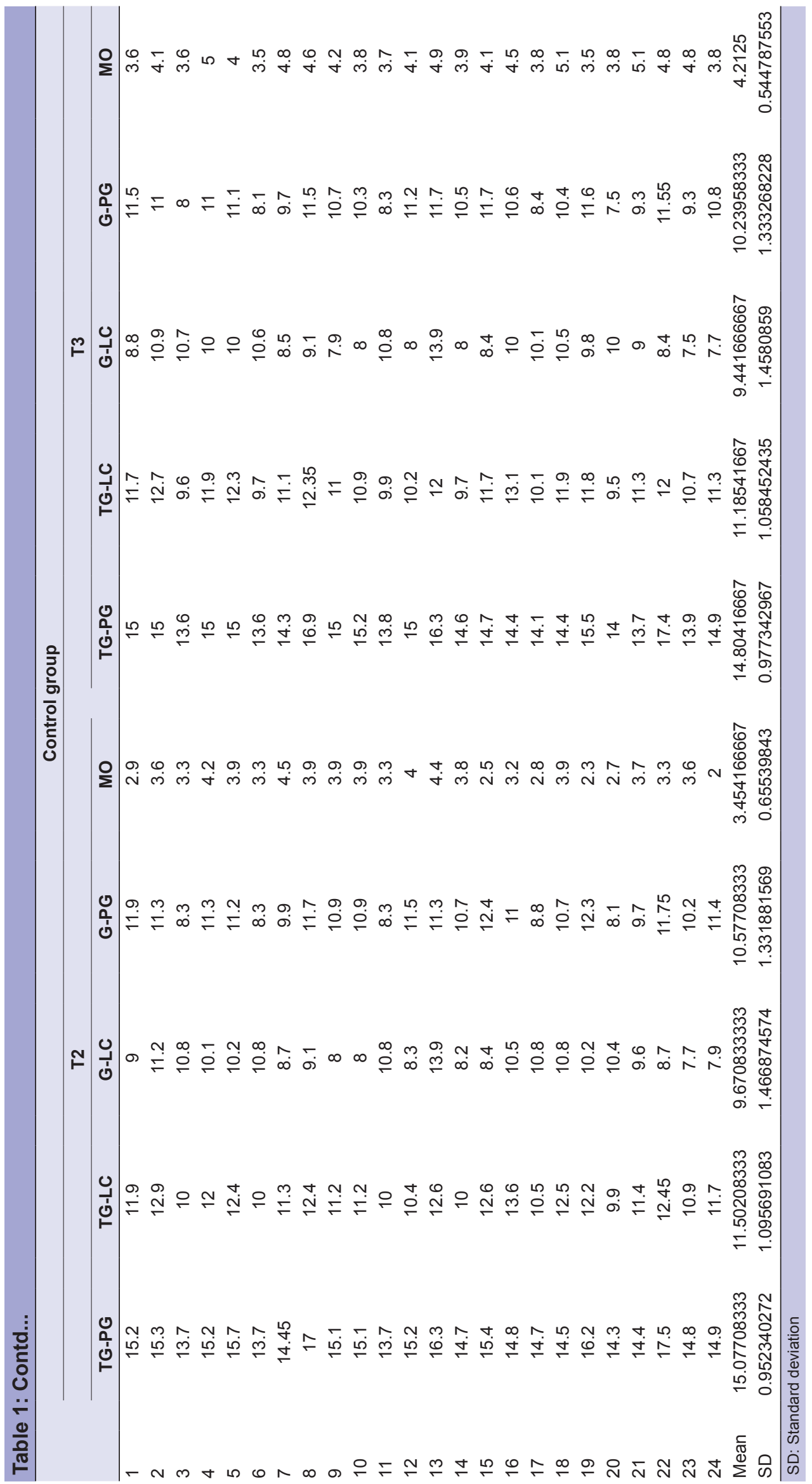


Oteri, et al.: ENF outcome on impacted lower third molar surgery

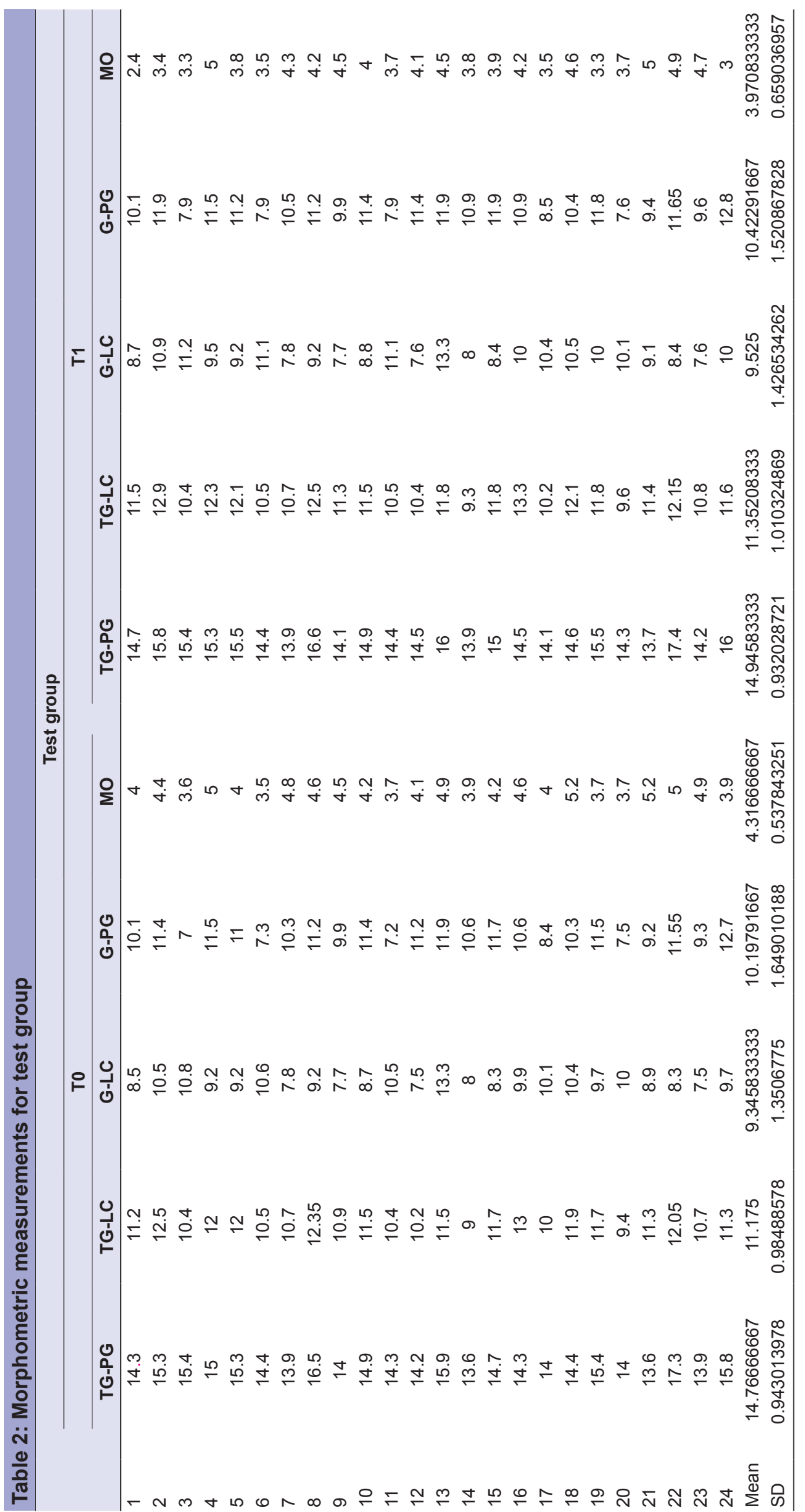


Oteri, et al.: ENF outcome on impacted lower third molar surgery

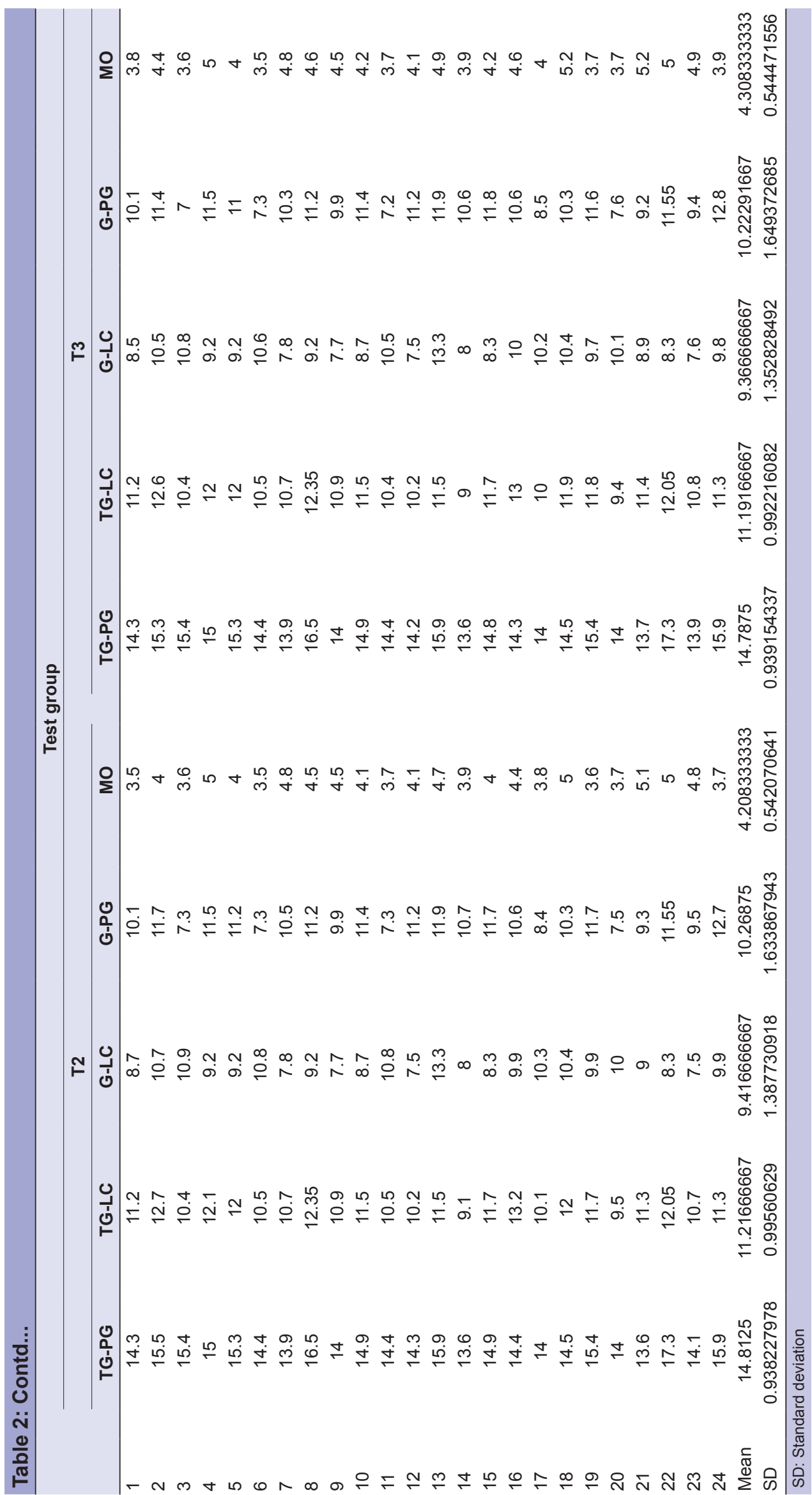




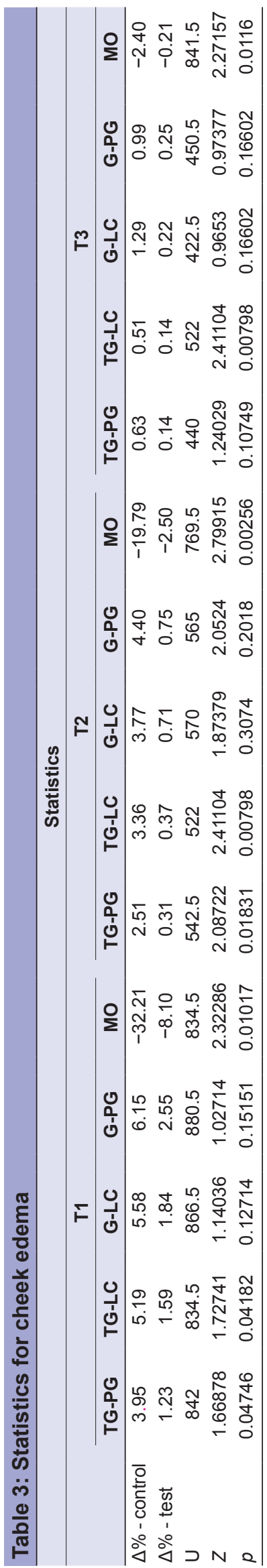

It refers to different noninvasive, nonpharmacological treatments, in which pain relief is achieved through both central and peripheral mechanisms. The TENS device was proved to be easy-to-use, with a safety profile. No adverse events related to the ENF occurred during the study. If on the one hand, ENF represents a solution for all those patients with allergies and intolerances to analgesics drugs on the other side medical technology has significant financial implications despite the savings for the prescriptions of painkillers.

Consequently, this study is added to others in a constant effort to explore whether medical technology may result in a measurable therapeutic benefit (e.g., quality of life) leaving aside cost-effectiveness evaluation. ${ }^{[31]}$

Future research direction may be the investigation of others biochemical parameters looking for a laboratory evidence of the anti-inflammatory effect of ENF which is reflected on the change in the values of expression of evaluated markers.

\section{CONCLUSIONS}

This study explored the role of TENS treatment in the postop outcome of impacted lower third molar surgery, a condition frequently observed among the general adult population. TENS is a widely used, noninvasive, nonpharmacological treatment that may grant pain reduction through different mechanisms. ENF is a portable TENS device, and it was proved to be reliable and easy-to-use. TENS indeed lowered the postsurgical pain and the edema and improved the functional recovery in the means of the stomatognathic apparatus. Therefore, limiting the painkillers consumption the days following surgery, TENS should be considered as an alternative in the management of the clinical outcome of invasive oral procedures, with its only drawback being the request of attendance for the treatment. Further studies may investigate other inflammatory markers to clarify whether or not TENS has a role in reducing inflammatory response.

\section{Declaration of patient consent}

The authors certify that they have obtained all appropriate patient consent forms. In the form the patient(s) has/have given his/her/their consent for his/her/their images and other clinical information to be reported in the journal. The patients understand 
Oteri, et al.: ENF outcome on impacted lower third molar surgery

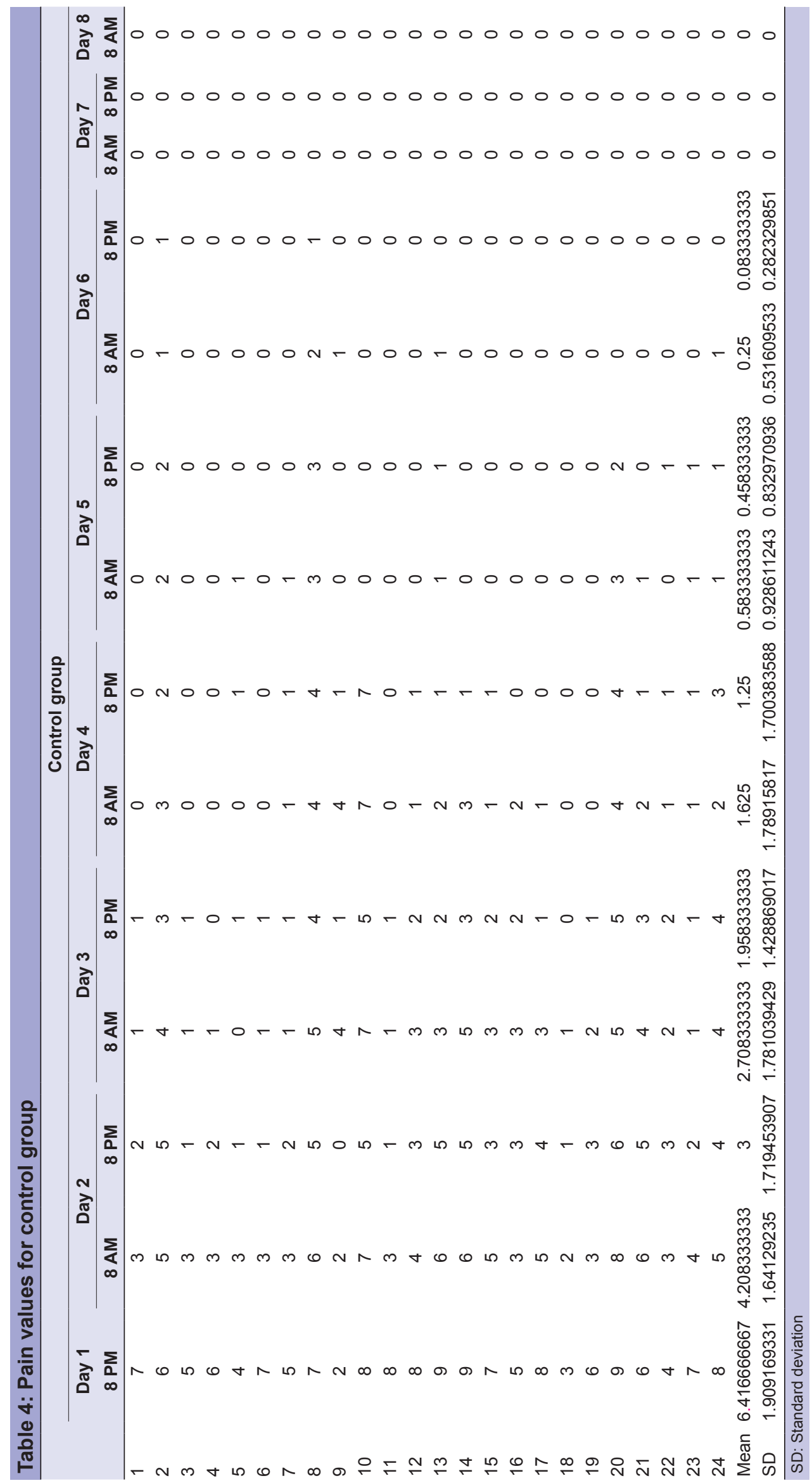


Oteri, et al.: ENF outcome on impacted lower third molar surgery

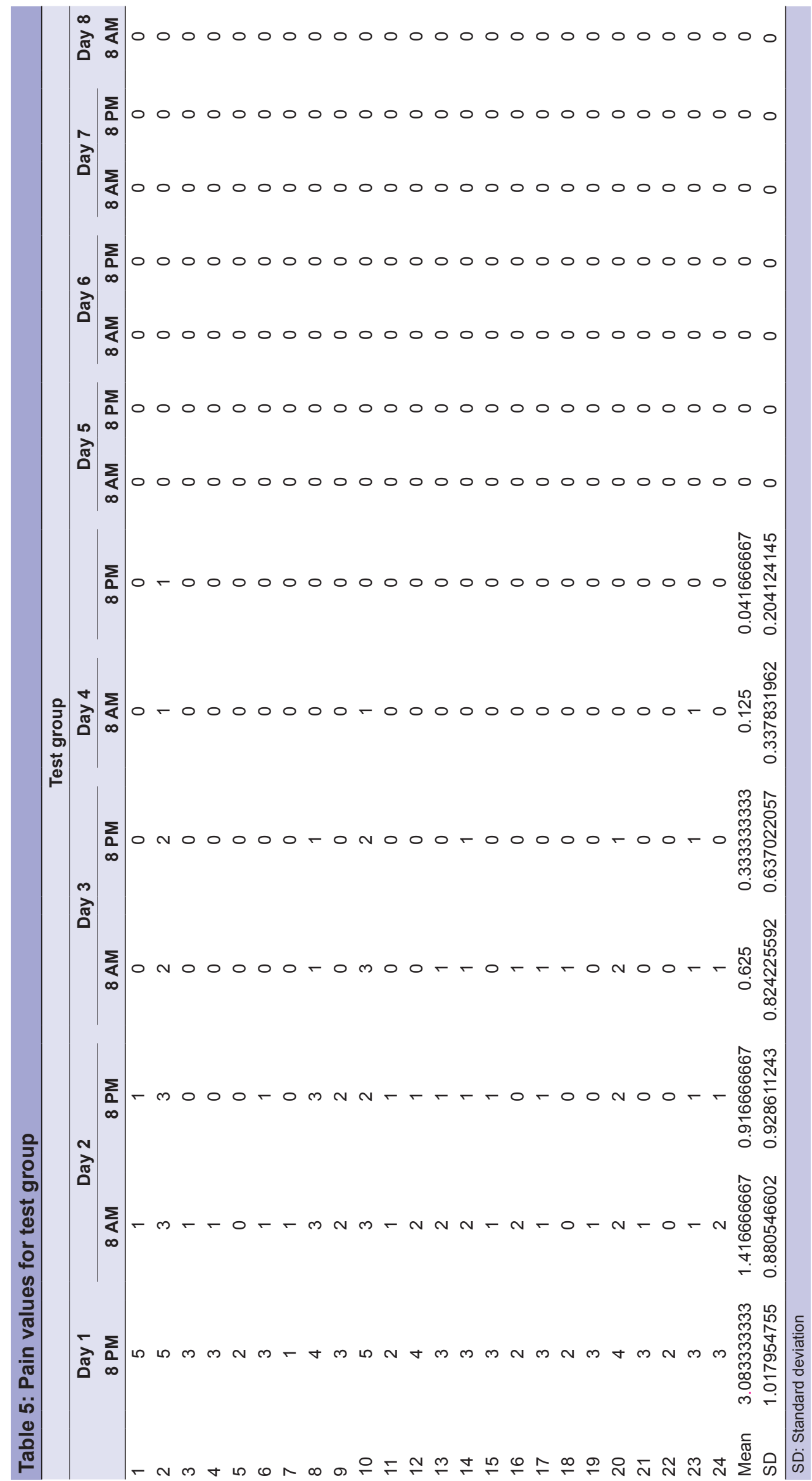


Oteri, et al.: ENF outcome on impacted lower third molar surgery

\begin{tabular}{|c|c|c|c|c|c|c|c|c|c|c|c|}
\hline & \multirow{2}{*}{$\begin{array}{l}\text { Day } 1 \\
8 \text { PM }\end{array}$} & \multicolumn{2}{|c|}{ Day 2} & \multicolumn{2}{|c|}{ Day 3} & \multicolumn{2}{|c|}{ Day 4} & \multicolumn{2}{|c|}{ Day 5} & \multicolumn{2}{|c|}{ Day 6} \\
\hline & & 8 AM & 8 PM & $8 \mathrm{AM}$ & 8 PM & $8 \mathrm{AM}$ & 8 PM & $8 \mathrm{AM}$ & 8 PM & $8 \mathrm{AM}$ & 8 PM \\
\hline $\bar{U}$ & 46 & 28 & 88.5 & 80 & 75 & 117 & 117.5 & 180 & 204 & 228 & 264 \\
\hline$Z$ & 4.97965 & 5.3508 & 4.10331 & 4.27858 & 4.38168 & 3.51565 & 3.50534 & 2.21661 & 1.72174 & 1.22687 & 0.48456 \\
\hline$p$ & 0.000001 & 0.000001 & 0.000001 & 0.000001 & 0.000001 & 0.00022 & 0.00022 & 0.01321 & 0.04272 & 0.10935 & 0.31561 \\
\hline
\end{tabular}

that their names and initials will not be published and due efforts will be made to conceal their identity, but anonymity cannot be guaranteed.

\section{Financial support and sponsorship Nil.}

\section{Conflicts of interest}

There are no conflicts of interest.

\section{REFERENCES}

1. Jones I, Johnson MI. Transcutaneous electrical nerve stimulation. Contin Educ Anaesth Crit Care Pain 2009:9:130-5.

2. Sluka KA, Walsh D. Transcutaneous electrical nerve stimulation: Basic science mechanisms and clinical effectiveness. J Pain 2003;4:109-21.

3. Walsh DM, Baxter D. Transcutaneous electrical nerve stimulation (TENS): A review of experimental studies. Eur J Phys Med Rehabil 1996;6:42-50.

4. Melzack R, Wall PD. Pain mechanism: A new theory. Science 1965;150:971-9.

5. Nnoaham KE, Kumbang J. Transcutaneous electrical nerve stimulation (TENS) for chronic pain. Cochrane Database Syst Rev 2008;(3):CD003222.

6. Katch EM. Applications of transcutaneous electrical nerve stimulation in dentistry. Anesth Prog 1986;33:156-60.

7. Sbruzzi G, Silveira SA, Silva DV, Coronel CC, Plentz RD. Transcutaneous electrical nerve stimulation after thoracic surgery: Systematic review and meta-analysis of 11 randomized trials. Rev Bras Cir Cardiovasc 2012;27:75-87.

8. Hurlow A, Bennett MI, Robb KA, Johnson MI, Simpson KH, Oxberry SG, et al. Transcutaneous electric nerve stimulation (TENS) for cancer pain in adults. Cochrane Database Syst Rev 2012;3:CD006276.

9. Walsh DM, Howe TE, Johnson MI, Sluka KA. Transcutaneous electrical nerve stimulation for acute pain. Cochrane Database Syst Rev 2009;2:CD006142

10. Khadilkar A, Odebiyi DO, Brosseau L, Wells GA. Transcutaneous electrical nerve stimulation (TENS) versus placebo for chronic low-back pain. Cochrane Database Syst Rev 2008;4:CD003008.

11. Rutjes AW, Nüesch E, Sterchi R, Kalichman L, Hendriks E, Osiri M, et al. Transcutaneous electrostimulation for osteoarthritis of the knee. Cochrane Database Syst Rev 2009;4:CD002823.

12. Kugelberg CF. Impacted lower third molars and periodontal health. An epidemiological, methodological, retrospective and prospective clinical, study. Swed Dent J Suppl 1990;68:1-52.

13. Chiapasco M, Crescentini M, Romanoni G. Germectomy or delayed removal of mandibular impacted third molars: The relationship between age and incidence of complications. J Oral Maxillofac Surg 1995;53:418-22.

14. Nemcovsky CE, Libfeld H, Zubery Y. Effect of non-erupted $3^{\text {rd }}$ molars on distal roots and supporting structures of approximal teeth A radiographic survey of 202 cases. J Clin Periodontol 1996;23:810-5.

15. Menditti D, Laino L, Cicciù M, Mezzogiorno A, Perillo L, Menditti M, et al. Kissing molars: Report of three cases and new prospective on aetiopathogenetic theories. Int J Clin Exp Pathol 2015;8:15708-18.

16. Laino L, Menditti D, Lo Muzio L, Laino G, Lauritano F, Cicciù M, et al. Extraoral surgical approach of ectopic mandibular third molar to the lower border of mandible. J Craniofac Surg 2015;26:e256-60.

17. Chiapasco M, De Cicco L, Marrone G. Side effects and complications associated with third molar surgery. Oral Surg Oral Med Oral Pathol 1993;76:412-20.

18. Pell GJ, Gregory BT. Impacted mandibular third molars: Classifications and modified techniques for removal. Dent Dig 1933;39:330.

19. Oteri G, Cicciù M, Peditto M, Catalano A, Loddo S, Pisano M, et al. Does Vitamin D3 have an impact on clinical and biochemical parameters related to third molar surgery. J Craniofac Surg 2016;27:469-76.

20. Oztas N, Olmez A, Yel B. Clinical evaluation of transcutaneous electronic nerve stimulation for pain control during tooth preparation. Quintessence Int 1997;28:603-8.

21. Varrese M, Guerrini G. Approach to electrical anesthesia: Nine years of experience in odontostomatological surgery. Anesth Prog 1992;39:185.

22. Yameen F, Shahbaz NN, Hasan Y, Fauz R, Abdullah M. Efficacy of transcutaneous electrical nerve stimulation and its different modes in patients with trigeminal neuralgia. J Pak Med Assoc 2011;61:437-9.

23. Steller M, Chou L, Daniels TE. Electrical stimulation of salivary flow in patients with Sjögren's syndrome. J Dent Res 1988;67:1334-7.

24. Ghensi P, Cucchi A, Creminelli L, Tomasi C, Zavan B, Maiorana C, et al. Effect of oral administration of bromelain on postoperative discomfort after third molar surgery. J Craniofac Surg 2017;28:e191-7.

25. Lima TC, Bagordakis E, Falci SG, Dos Santos CR, Pinheiro ML. Pre-emptive effect of dexamethasone and diclofenac sodium associated with codeine on pain, swelling, and trismus after third molar surgery: A split-mouth, randomized, triple-blind, controlled clinical trial. J Oral Maxillofac Surg 2018;76:60-6.

26. Osunde OD, Anyanechi CE, Bassey GO. Prevention of alveolar osteitis after third molar surgery: Comparative study of the effect of warm saline and chlorhexidine mouth rinses. Niger J Clin Pract 2017;20:470-3.

27. Chen Q, Chen J, Hu B, Feng G, Song J. Submucosal injection of dexamethasone reduces postoperative discomfort after third-molar extraction: A systematic review and meta-analysis. J Am Dent Assoc 2017;148:81-91.

28. Bamgbose BO, Akinwande JA, Adeyemo WL, Ladeinde AL, Arotiba GT, Ogunlewe MO, et al. Effects of co-administered dexamethasone and diclofenac potassium on pain, swelling and trismus following third molar surgery. Head Face Med 2005;1:11.

29. Sortino F, Messina G, Pulvirenti G. Evaluation of postoperative mucosa and skin temperature after surgery for impacted third molar. Minerva Stomatol 2003;52:393-9.

30. Markovic A, Todorovic LJ. Effectiveness of dexamethasone and low-power laser in minimizing oedema after third molar surgery: A clinical trial. Int J Oral Maxillofac Surg 2007;36:226-9.

31. Sorenson C, Drummond M, Bhuiyan Khan B. Medical technology as a key driver of rising health expenditure: Disentangling the relationship. Clinicoecon Outcomes Res 2013;5:223-34 